\title{
BMJ Open Appropriateness of cases presenting in the emergency department following ambulance service secondary telephone triage: a retrospective cohort study
}

Kathryn Eastwood, ${ }^{1,5}$ Karen Smith, ${ }^{1,5}$ Amee Morgans, ${ }^{2}$ Johannes Stoelwinder ${ }^{1,2}$

To cite: Eastwood K, Smith K, Morgans A, et al. Appropriateness of cases presenting in the emergency department following ambulance service secondary telephone triage: a retrospective cohort study. BMJ Open 2017;7:e016845. doi:10.1136/ bmjopen-2017-016845

- Prepublication history for this paper is available online To view these files, please visit the journal online (http://dx.doi. org/10.1136/bmjopen-20170168454).

Received 18 March 2017 Revised 11 September 2017 Accepted 12 September 2017

CrossMark

${ }^{1}$ Department of Epidemiology and Preventive Medicine,

Monash University, Melbourne, Victoria, Australia

${ }^{2}$ Emergency Services

Telecommunications Authority,

Melbourne, Victoria, Australia

${ }^{5}$ Ambulance Victoria, Melbourne, Victoria, Australia

Correspondence to

Kathryn Eastwood;

eastwood@monash.edu

\section{ABSTRACT}

Objective To investigate the appropriateness of cases presenting to the emergency department (ED) following ambulance-based secondary telephone triage.

Design A pragmatic retrospective cohort analysis of all the planned and unplanned ED presentations within 48 hours of a secondary telephone triage.

Setting The secondary telephone triage service, called the Referral Service, and the hospitals were located in metropolitan Melbourne, Australia and operated 24 hours a day, servicing 4.25 million people. The Referral Service provides an in-depth secondary triage of cases classified as low acuity when calling the Australian emergency telephone number.

Population Cases triaged by the Referral Service between September 2009 and June 2012 were linked to ED and hospital admission records ( $\mathrm{N}=44,523)$. Planned $\mathrm{ED}$ presentations were cases referred to the ED following the secondary triage, unplanned ED presentations were cases that presented despite being referred to alternative care pathways.

Main outcome measures Appropriateness was measured using an ED suitability definition and hospital admission rates. These were compared with mean population data which consisted of all of the ED presentations for the state (termed the 'average Victorian ED presentation').

Results Planned ED presentations were more likely to be ED suitable than unplanned ED presentations (OR 1.62; $95 \% \mathrm{Cl} 1.5$ to $1.7 ; \mathrm{p}<0.001)$ and the average Victorian ED presentation (OR 1.85; $95 \% \mathrm{Cl} 1.01$ to $3.4 ; \mathrm{p}=0.046$ ). They were also more likely to be admitted to the hospital than the unplanned ED presentation (OR 1.5; 95\% $\mathrm{Cl} 1.4$ to 1.6; $\mathrm{p}<0.001$ ) and the average Victorian ED presentation (OR $2.3,95 \% \mathrm{Cl} 2.24$ to 2.33 ; $\mathrm{p}<0.001$ ). Just under $15 \%$ of cases diverted away from the emergency care pathways presented in the ED (unplanned ED attendances), and $9.5 \%$ of all the alternative care pathway cases were classified as ED suitable and $6.5 \%$ were admitted to hospital.

Conclusions Secondary telephone triage was able to appropriately identify many ED suitable cases, and while most cases referred to alternative care pathways did not present in the ED. Further research is required to establish that these were not inappropriately triaged away from the emergency care pathways.
Strengths and limitations of this study

- This is the first Australian study to link secondary telephone triage records to emergency department (ED) and hospital records to track a patient's process through the prehospital to hospital healthcare system.

- This is the first large-scale study to investigate the appropriateness of cases presenting in the ED following secondary telephone triage.

- This study did not rely on retrospective expert opinion to measure appropriateness but used a range of independently derived ED outcomes to assess appropriateness.

- Due to the heterogeneity of ambulance services and secondary telephone triage services, the generalisability of the results may be limited; however, the methodology can be replicated to generate locally reproducible results.

\section{INTRODUCTION}

An increasing proportion of ambulance service workload involves patients with low-acuity health events that do not require the specific resources provided by ambulance services or emergency departments (ED). ${ }^{1-19}$ Responding to these cases with a traditional emergency ambulance attendance and transport to a hospital ED negatively impacts on ambulance services' efficiency and efficacy by reducing the availability of these resources for emergency cases and thus potentially compromising patient outcomes. ${ }^{8142021}$ The notion of whether these unnecessary ED users place a similar stress on the ED is one of the contentions, with some research suggesting that the number and the impact of these patients is much lower than the high levels reported in other literature. ${ }^{22-24}$ Depending on the study, these figures range from as little as $5 \%$ up to $82 \%$ of all ED presentations. ${ }^{22} 2425$ Despite this, there appears to be some level of consensus that these patients often present with conditions that can be suitability 
managed in community-based healthcare services rather than the ED. ${ }^{1521}$ The ability of ambulance services and EDs to expand resources to meet their increasing demand is limited, and as a result, alternative strategies are being implemented to manage low-acuity cases. ${ }^{26-37}$

Secondary telephone triage has been used by some ambulance services as a demand management strategy for the identification and referral of low-acuity cases to alternative healthcare services and away from the emergency care pathways involving ambulances and the ED. ${ }^{138}$ As its name implies, secondary telephone triage occurs after a primary triage has taken place when a patient contacts the emergency dispatch centre. Cases classified as low acuity during primary triage are then triaged by qualified nurses or paramedics to further elucidate the patients presenting problem. Where appropriate these cases are diverted to other means of transportation to hospital, alternative service providers for management outside of the emergency care pathways, or they are given self-care advice for management in the home. Ambulance Victoria in Victoria, Australia, operates the Referral Service, a secondary telephone triage service that managed nearly $12 \%$ of the total emergency ambulance workload in the capital city of Melbourne between 2009 and 2012. The Referral Service diverted $72.4 \%$ of the triaged low-acuity cases away from emergency ambulances and $32.2 \%$ away from the ED. ${ }^{1}$ This strategy has had a measurable impact in metropolitan Melbourne and across Victoria with a $10 \%$ decrease in growth of demand for emergency ambulance transports on its implementation. ${ }^{39}$

Despite the policy intention of reducing low-acuity cases from the emergency ambulance and from ED workloads, some cases remain or re-emerge in the emergency care pathways following secondary triage. ${ }^{140}$ These can be categorised into two groups of casesthose that are planned ED attendances and those that are unplanned ED attendances. Planned ED attendances are cases identified at secondary telephone triage as suitable to remain in the emergency care pathways. These cases may be sent an emergency ambulance, non-emergency ambulance or referred to self-present at the ED. ${ }^{1}$ If these cases are later identified as inappropriate for the $\mathrm{ED}$, then the question is raised about whether they were incorrectly triaged by the Referral Service to these care pathways. Unplanned ED attendances are cases that present in the ED despite being referred to alternative care pathways. These pathways include advice to allow the patient to manage their presenting problem at home (self-care advice), referral to the patient's own general practitioner (GP) or allied healthcare worker or referral to one of a range of alternate service providers contracted by Ambulance Victoria, who will attend the patient's home. ${ }^{1}$ If these cases subsequently and appropriately attend the ED, they may represent a cohort of cases that were incorrectly triaged by the Referral Service as suitable for alternative care pathways.

The effectiveness of an ambulance-based secondary telephone triage service is reflected in its ability to provide patients with the most appropriate care for their needs. The appropriateness of the ED presentation of cases following secondary telephone triage has only been investigated in two small trials which found that patients were more likely to be admitted to the hospital if they were identified as being suitable to remain in the emergency care pathways (ie, they were a planned ED attendance).$^{30-32}$ No large-scale evaluations have been conducted using an established secondary telephone triage service operating within an ambulance service.

The aim of this study was to investigate the appropriateness of the ED presentation of cases following secondary telephone triage by the Referral Service.

\section{METHODS \\ Design}

A pragmatic retrospective cohort analysis was conducted of all the planned and unplanned ED presentations within the emergency care and alternative care pathways within 48 hours of a Referral Service triage.

\section{Setting}

Ambulance Victoria is a statewide publicly funded ambulance service operating in the state of Victoria, Australia. In June 2012, 4.25 million people lived in metropolitan Melbourne which covers an area of approximately $10000 \mathrm{~km}^{2}{ }^{41}$ During the study timeframe the Referral Service operated within metropolitan Melbourne 24 hours a day, 7 days a week.

The Referral Service has been described extensively elsewhere. ${ }^{1}$ Briefly, cases identified as low acuity during the call to the emergency services telephone number (in Australia, this is triple zero), using the Advanced Medical Priority Dispatch System, are transferred for secondary triage. Case-types designated as low acuity have been specifically identified by Ambulance Victoria as having low paramedic treatment and transportation rates and are unlikely to represent to the ambulance service within a 24 hours timeframe. Referral Service call-takers use a condition-specific computer-based questioning algorithm (Care Enhanced Call Centre) ${ }^{42}$ during secondary telephone triage to arrive at a disposition with a recommended resource allocation outcome as listed below.

\section{Emergency care pathways}

- Return for emergency ambulance dispatch;

- Non-emergency ambulance dispatch;

- Advise the patient to self-present at the ED.

\section{Alternative care pathways}

- Referral to an Alternative Service Provider;

- Self-care advice including home care or to seek further non-urgent medical attention independently (figure 1). 


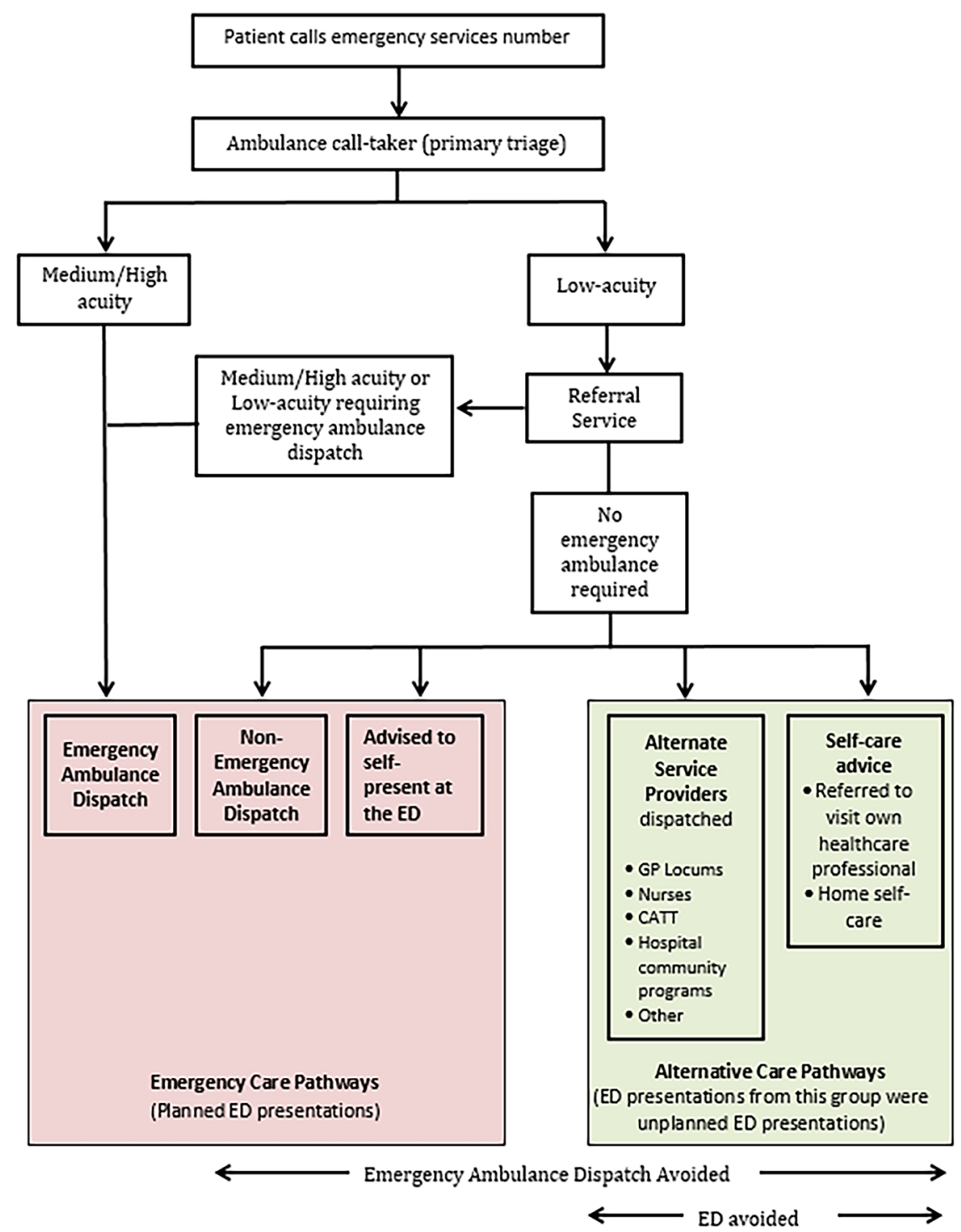

Figure 1 Case flow from the call to the emergency services to Referral Service outcome. CATT, crisis assessment and treatment teams; ED, emergency department; GP, general practitioner.

The alternative service providers that the Referral Service uses include out-of-hours home-visiting doctor services, home-visiting nurses, hospital outreach programme (that send allied health staff into the community), crisis assessment and treatment teams for psychiatric cases, poisons telephone advice line and other services that can assist with non-medical issues such as lifting patients.

\section{Data sources}

Data were collected between September 2009 and June 2012 for the datasets below unless otherwise stated.

\section{Referral Service}

Referral Service records were extracted from the Referral Service database. Data items included case date and time, case number, deidentified patient-specific code, date of birth, age, gender, suburb, presenting problem, free text entry with details of the patient triage and triage disposition.

\section{Paramedic records}

Cases referred for an emergency ambulance dispatch had an electronic patient care record (paramedic record) generated documenting assessment, treatment, demographic and operational information. Paramedic records included case date and time, case number, Medicare suffix (first three characters of the patients given name), date of birth, age, gender, suburb, dispatch urgency, treatment, transport outcome, destination hospital (where appropriate) and transport urgency (where appropriate).

\section{Hospital datasets (ED and admission records)}

Hospital data were sourced from the Victorian Emergency Minimum Dataset (ED records) and the Victorian Admitted Episode Dataset (admission records). The ED records contains de-identified administrative, demographic, treatment and clinical information detailing ED presentations at designated Victorian public hospitals 


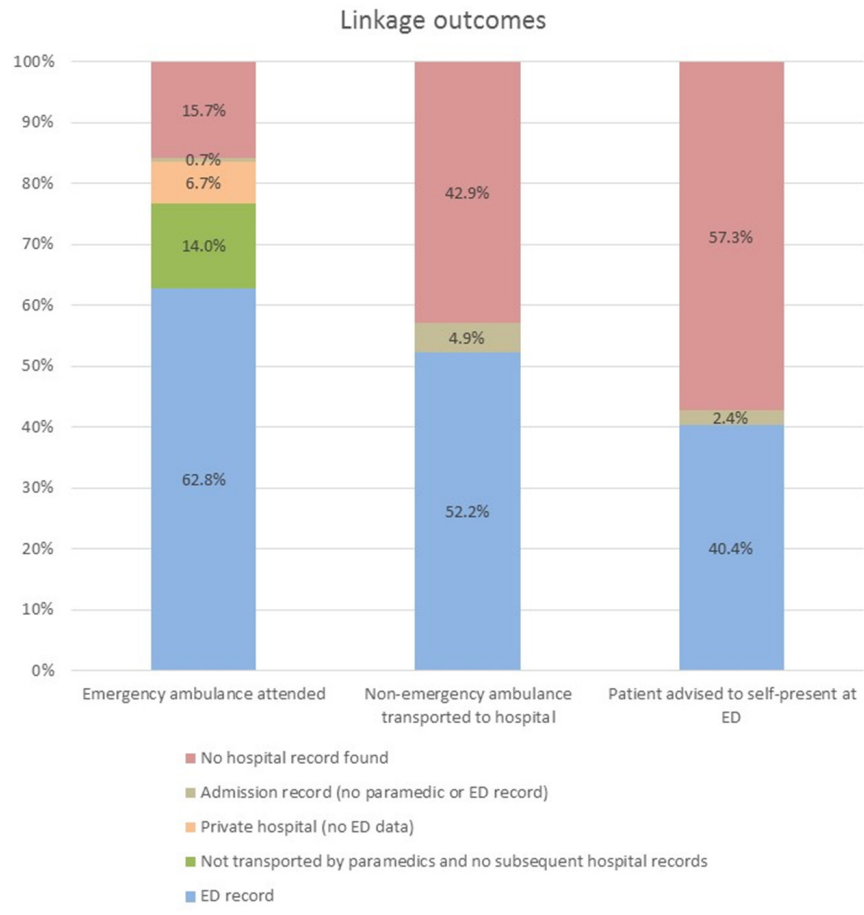

Figure 2 Linkage outcomes for each of the emergency care pathways. ED, emergency department.

and others as directed by the Victorian Government Department of Health. ${ }^{43}$ Similarly, the admission records contains deidentified administrative data for Victorian hospital admissions. ${ }^{44}$ The Department of Health does not routinely collect ED data from private hospitals (privately owned hospitals running on a user-pays system), which on average received about $8.1 \%$ of all Victorian ED presentations. ${ }^{25}{ }^{45}$ Private hospitals do provide their admission records to the Department of Health and this was the only indicator of whether a patient attended a private hospital ED. If, however, a patient was not admitted following their ED presentation at a private hospital, then no record of their ED presentation could be obtained. Variables extracted included case date and time, deidentified patient-specific code (this is a different code to that used in the Referral Service dataset), International Classification of Diseases, 10th Edition, Australian Modification code, arrival mode, ED triage category, outgoing referral, admission and death.

\section{Data linkage}

Deterministic data linkage was used to link the Referral Service and paramedic records for cases referred to the emergency ambulance pathway (the ambulance datasets). ${ }^{46}$ The variables used for linkage included case date, case number, date of birth, age, gender and suburb. Nearly all of the paramedic records $(94.7 \%)$ were linked to Referral Service records, and these linkages were verified using case-time, presenting problem, urgency level set by Referral Service call-takers and free-text analysis where required. This process resulted in seven linkages that could not be verified as a true match $(0.0003 \%$ error rate).
These ambulance datasets were then linked to the hospital datasets (the ED and admission records) also using deterministic data linkage methods. ${ }^{46}$ For this linkage ambulance case number, Medicare suffix, date of birth, address (postal code or locality), and record date within 48 hours of arrival at the ED were used. The algorithm used allowed for a single day discrepancy in date of birth, date of ambulance records and date of hospital records. Validation of the deterministic linkage between the linked ambulance datasets and the hospital datasets was completed using gender. A mismatch was identified for $2 \%$ of linkages and these were discarded $(n=856)$. Linkages where the hospital record occurred before Referral Service triage were also discarded $(n=2300)$.

\section{Data linkage outcomes for planned ED presentations}

During the study timeframe, $27.5 \%$ of all metropolitan Ambulance Victoria cases that had an ambulance attendance were not transported to hospital. This, combined with the fact that the private hospitals do not supply their ED records, meant a linkage rate of $100 \%$ between ambulance and hospital records was not expected.

Figure 2 depicts the proportion of Referral Service cases for each of the three emergency care pathways for which an ED record was linked. Cases in the emergency ambulance pathway had the highest rate of linkage to ED records $(62.8 \%)$. Some cases in this pathway were found to have been transported to private hospital (6.7\%), meaning no ED record was available, or left at home after paramedic assessment (14.0\%). The remaining $15.7 \%$ of cases for which an ED record was expected were unable to be accounted for.

Over half of the 'non-emergency ambulance' pathway cases $(57.3 \%)$ and $42.8 \%$ of the 'self-present at ED' pathway cases were linked to an ED record or an admission record (figure 2). Some of these cases may have been transported to a private hospital. The proportion of private hospital ED presentations is $8.1 \%$ of all Victorian ED presentations, and assuming a similar proportion of this population attended a private hospital ED, a large number of cases would remain unaccounted for.

The lack of an ED record for $37.0 \%$ of the planned ED attendances does not necessarily mean these patients did not attend the ED. The linkage process may have failed to identify a corresponding ED record, or they may have attended a private hospital ED. When comparing the number of cases Ambulance Victoria reported as being transported to hospital, to the number of ambulance presentations reported in the Australian government reports, ${ }^{25} 4748$ there is only a $2.2 \%$ discrepancy in the numbers. This suggests that there may be a number of missed linkages rather than simply no presentation at the $\mathrm{ED}$, however a level of non-compliance was expected. ${ }^{49}$

A systematic bias evaluation was conducted, comparing age, gender and main presenting problems between the cases with a linked ED record and those with no linked ED record. Significance testing was pragmatically unsuitable because the large size of the dataset would result 
in a high level of statistical sensitivity to small distribution differences. This is demonstrated in table 1 , where the gender distribution for the 'ED record' and 'no ED record' group was minimal $(54.3 \%$ vs $56.1 \%)$, and the mean age for the non-emergency ambulance records only varied by 1 year, yet the significance testing found these to be significant differences between these groups. When comparing the presenting problems of the cases within each group in table 1 , there was little variation in the three most common case types between those with and without an ED record. Therefore, age, gender and presenting problem were considered as not imposing any clinically significant bias on the results, and the results presented in this paper were considered to be representative of the cases referred to the emergency care pathways by the Referral Service.

\section{Patient involvement}

This was a retrospective study of established data sources, as such no patients were involved in this study.

\section{Patient outcomes}

General demographic, triage outcome and main presenting problem information was collected during this study.

\section{Indicators of appropriateness}

ED suitability and admission to hospital were used as indicators of appropriateness for cases that presented at the ED. Planned and unplanned ED presentation were analysed using these measures and then compared with the average Victorian ED presentation.

\section{ED suitability}

ED suitability was based on a modified version of the 'potentially avoidable GP-type presentation' measure used by the Australian Government for ED presentations that are considered avoidable had an appropriate community-based service been accessed ${ }^{50} \mathrm{~A}$ 'potentially avoidable GP-type presentation' is defined as cases that present to an ED where the patient:

- was triaged as a category 4 or 5 according to the Australian Triage Scale ${ }^{51}$;

- did not arrive by ambulance;

- was not admitted to the hospital, referred to another hospital,

- did not die. ${ }^{50}$

This 'potentially avoidable GP-type presentation' outcome was modified in this study to exclude the criterion involving arrival by ambulance and was referred to as 'ED suitability'.

\section{Hospital admission}

Despite hospital admission being used as part of the ED suitability indicator, this indicator has also been used in isolation in other studies ${ }^{30} 32$ and was therefore retained to allow for comparison. Also, hospital admission was provided by both public and private hospitals, therefore allowing for cases transported to private hospitals to be included in the analysis.

\section{Average Victorian ED presentation for Victoria}

Each year the Australian government report the overall rates of hospital admission and "potentially avoidable GP-type presentations' for all public hospital ED presentations in each state of Australia. ${ }^{50}$ The overall rates are inclusive of all ED attendances, including Referral Service cases that present at the ED. The rates of ED suitability and hospital admission were compared with the overall rates for Victoria, which were referred to as 'the average Victorian ED presentation' in this paper. The rates from the $2011 / 2012$ report were used in this study. ${ }^{50}$

\section{Data analysis}

Data were analysed using descriptive statistics, $\chi^{2}$ tests of association, independent samples t-tests and logistic regressions to identify relationships with $95 \%$ CIs. All tests were considered to be significant at 0.05 level. All data analysis was performed using SPSS V.20. ${ }^{52}$

\section{RESULTS}

\section{Outcomes}

During the study timeframe, Ambulance Victoria received just over 1 million calls for assistance, of which $11.9 \%$ were triaged by the Referral Service. At the end of this triage, $69.5 \%$ of cases were referred to care pathways other than the emergency ambulance dispatch pathway, and 30.5\% were referred away from an ED presentation (the emergency care pathways). Figure 3 outlines the selection of cases eligible for inclusion in this study, resulting in 44523 cases undergoing further analysis.

\section{Patient demographics}

The gender distribution for cases presenting to the ED was similar for all care pathway groups (table 2). Triage outcomes that required the patients to self-source further care, including the 'self-present at the ED' cases and 'selfcare advice' cases, were younger than those sent further care (table 2).

Five main presenting problems made up $80 \%$ of the most common problems for each of the care pathways (table 2). These were abdominal pain, back pain, nausea and vomiting, urinary symptoms and dizziness and vertigo. Abdominal pain and back pain featured in the top five main presenting problems for every care pathway.

\section{ED suitability}

The planned ED presentations were more likely to be classified as ED suitable than the unplanned ED presentations (OR 1.62; 95\% CI 1.5 to $1.7 ; \mathrm{p}<0.001)$. The ED suitability for planned ED presentations ranged from $70.6 \%$ to $77.8 \%$ for each of the emergency care pathways, which was significantly higher than the ED suitability for the average Victorian ED presentations of $61.0 \%$ (OR 1.85; 95\% CI 1.01 to 3.4; $\mathrm{p}=0.046$ ) (Table 2). 


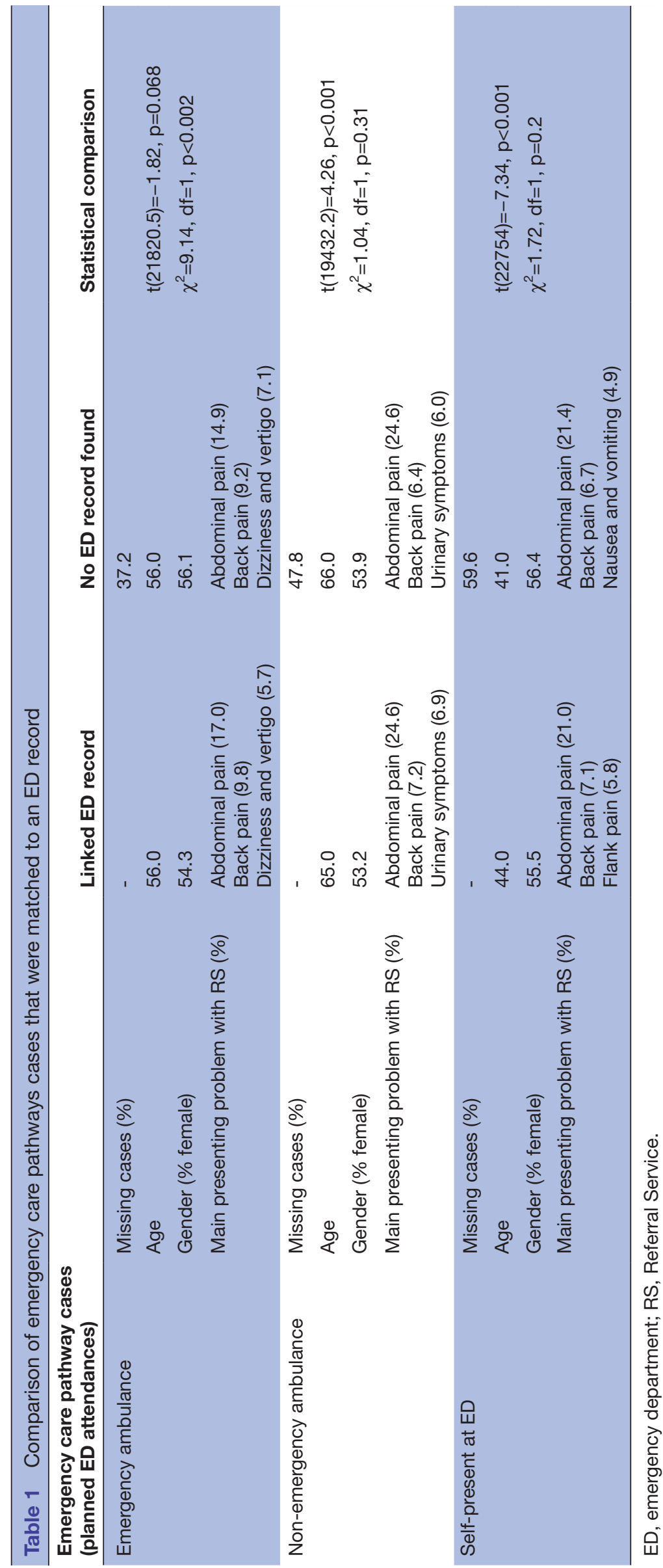

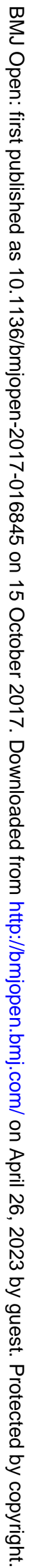




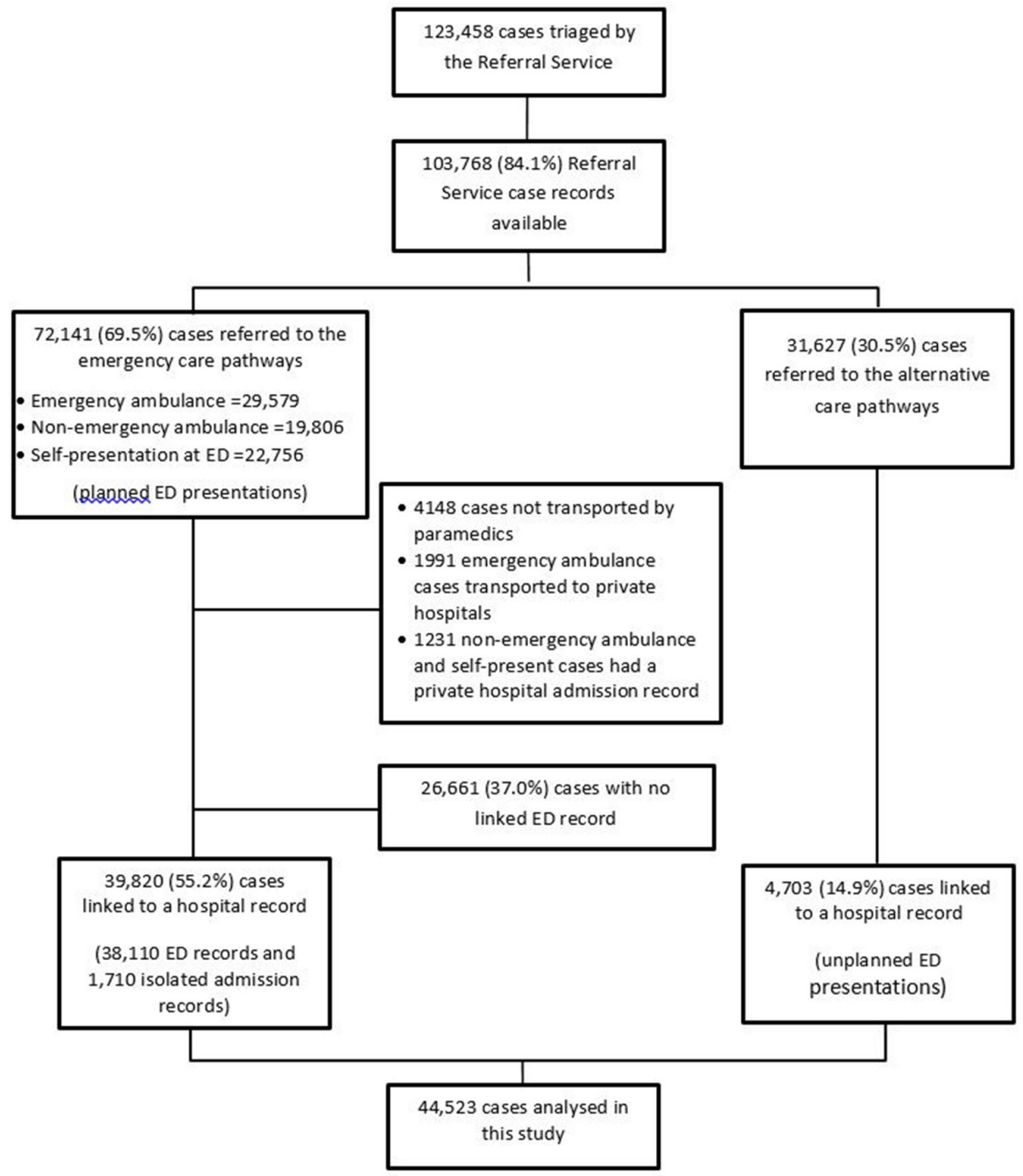

Figure 3 Selection planned and unplanned emergency department (ED) presentation cases for inclusion in this study.

Of the alternative care pathway cases, the unplanned ED presentations that were originally referred to alternative service providers had an ED suitability rate higher than the average Victorian ED presentation (68.8\%), and the cases originally given self-care advice had an ED suitability rate almost the same as the average Victorian ED presentation $(60.3 \%$ ) (table 2). These unplanned ED presentations were therefore at least as 'ED suitable' as the average Victorian ED presentation. It should be noted however, that only $19.3 \%$ of all the cases referred to the alternative service providers and $12.5 \%$ of all the cases given self-care advice presented in the ED. Overall, only $9.5 \%$ of the total alternative care pathway cases were identified as ED suitable $(14.7 \%$ of all the alternative service provider cases and $8.2 \%$ of all the self-care advice cases).

\section{Hospital admission}

Planned ED presentations were significantly more likely to be admitted to hospital than unplanned ED presentations $(53.8 \%$ vs $43.5 \%$; OR $1.5,95 \%$ CI 1.4 to 1.6 ; $\mathrm{p}<0.001)$. Both the planned ED presentations (OR 2.3, 95\% CI 2.24 to 2.33; $\mathrm{p}<0.001)$, and the unplanned ED presentations
(OR 1.6, 95\% CI 1.5 to $1.73 ; \mathrm{p}<0.001$ ) were more likely to be admitted than the average Victorian ED presentation $(36.0 \%$ ) (table 2$)$. Overall, only $6.5 \%$ of all the alternative care pathway cases were admitted to hospital ( $11.3 \%$ of all the alternative service provider pathway cases and $5.1 \%$ of all the self-care advice pathway cases).

\section{DISCUSSION}

This was the first large-scale study to link ambulance service data and hospital data to investigate the outcomes of both planned and unplanned ED presentations following an ambulance-based secondary telephone triage. Overall, the cases referred to the emergency care pathways (the planned ED presentations), appeared to be appropriate with ED suitability and hospital admission rates being higher than both the unplanned ED presentation group and the average Victorian ED presentation.

The decision to send cases to the alternative care pathways appears sound with over $85 \%$ not emerging in the emergency care system within 48 hours. The overall rates of ED suitability and admission for the cases sent 


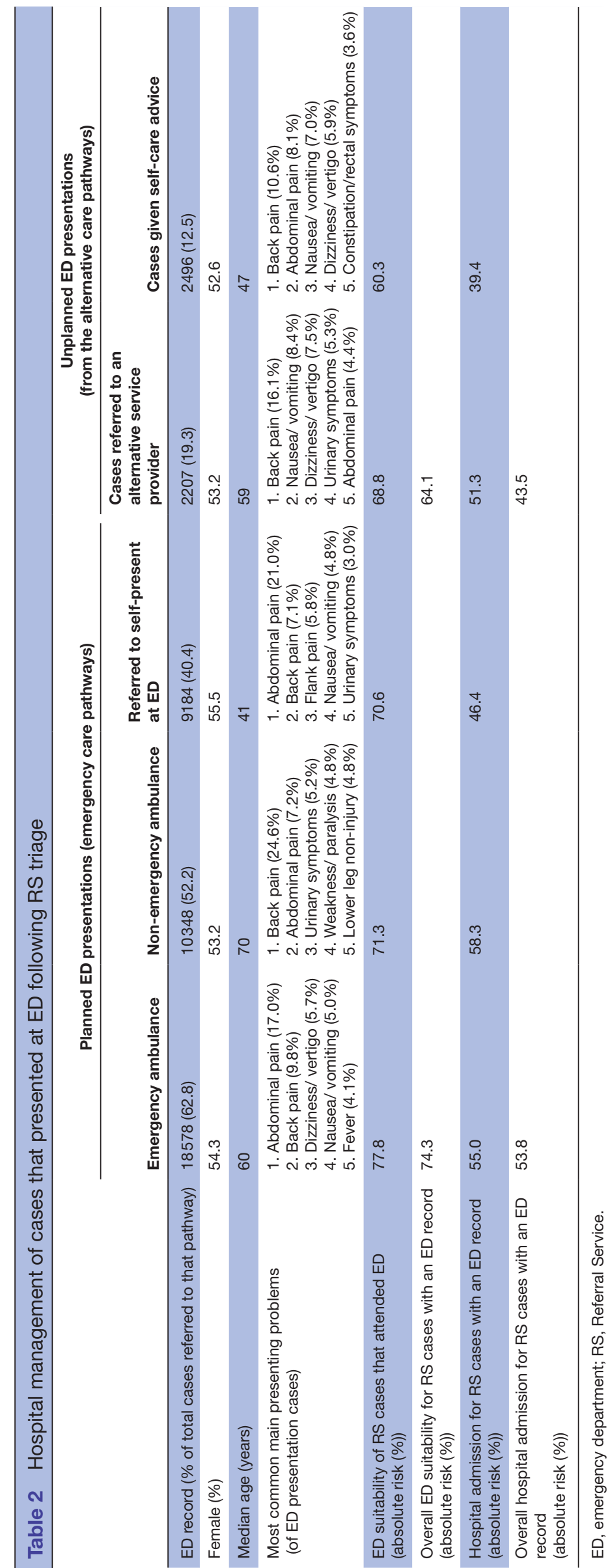

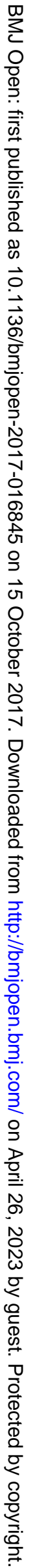


to the alternative care pathways were well below that of the average Victorian ED presentation predominantly because so few went on to present at the ED. When only the unplanned ED presentations were considered, the ED suitability and admission rates were the same, if not higher, that those for the average Victorian ED presentation. These results suggest that while the overall numbers of unplanned ED presentations were relatively small, they may have been appropriate for the ED and further investigation of these cases needs to be done to ensure they are not being incorrectly triaged to the alternative care pathways.

The results of this study are consistent with previous research, whereby cases classified as requiring an emergency ambulance were more likely to be admitted to the hospital than those classified as not requiring an emergency ambulance. ${ }^{30}$ The admission rate of cases in the alternative care pathways (unplanned ED presentations) of $6.5 \%$ was below that found in these other studies, which had rates of $9.2 \%$ and $15.8 \% .^{30}{ }^{32}$ This lower rate of admission may indicate that the secondary telephone triage process used by Ambulance Victoria, is more effective in identifying which cases are suitable for the alternative care pathways. While the previous research have accepted these admission rates and suggested the secondary telephone triage process is a safe and feasible means of managing ambulance demand, ${ }^{30} 32$ further investigation of the unplanned ED attendances is warranted.

This evaluation of ED suitability casts a broader net than simply basing the appropriateness of an ED presentation on whether a patient was admitted or not. The 'ED suitability' outcome measure increased the sensitivity, whereas the 'admissions only' outcome measure was felt to be more specific and prone to excluding appropriate cases. The ED suitability measure used a range of variables to eliminate the potential bias imposed by the decisions made by individual healthcare professionals during the patient care phase. Also, given that these variables are likely to be recorded in most emergency departments and are collected independent of any assessment of appropriateness, the ED suitability measure used in this study offers future researchers the opportunity to generate locally generalisable results that are also reproducible. ED treatment itself was not included in this outcome measure as it was the researcher's view that the ED healthcare workers will naturally instigate at a minimum, investigative procedures that could have been conducted in the primary care setting, which would have been viewed as a positive result for ED treatment. In this study, the ED suitability and admission outcome measures, also allowed for a comparison with the greater population of cases that present at the ED in Victoria.

While the results from this study suggest that the Referral Service was appropriate in filtering the cases ultimately destined for the ED, more can potentially be done to increase the sensitivity and specificity of the triage process. The unplanned ED presentation cases need to be further investigated to determine whether their condition evolved within the potential 48 hours window between Referral Service triage and ED presentation, whether they should have been triaged to the emergency care pathway, or whether other services, not within the suite of alternative service providers used by the Referral Service, would have been able to manage these cases in the primary care setting. Similarly, cases from the planned ED presentation pathway that were not ED suitable, or not admitted, need to be further investigated to determine if a primary care alternative is available to manage these cases out of the hospital setting.

Optimising the suite of pathways available to the Referral Service call-takers may lead to increased specificity of cases for emergency ambulance and the emergency department, therefore increasing the effectiveness of the Referral Service. In doing this, care should be taken to ensure that more than just physiological or clinical indicators are considered when decisions are made, particularly when these decisions result in the omission of a face-to-face assessment within a particular timeframe. Non-clinical situations have been identified where it would be considered appropriate for a low-acuity patient to be assessed and transported by paramedics, or present in the ED. ${ }^{53}$ An example of this is where there may be a perceived risk of physical harm to the patient, either through the threat of violence, an unattended minor or a patient who may appear physically or psychologically incompetent. ${ }^{53}$ Any secondary telephone triage process should ensure that the patients overall well-being is taken into consideration.

This study was limited by the inability to link some of the cases between the datasets. There are several possible reasons for a failure of an appropriate linkage, or for records to not have been available for linkage. These include private hospital attendance (therefore no ED records were available), transcription errors in case numbers and dates of birth during data acquisition and handovers, usage of a written paramedic record rather than an electronic paramedic record, ambulance cancellation prior to arrival and patient non-compliance. ${ }^{49}$ This highlights a need for consistent patient identifiers and a means of transcribing data at the various transitions of care that reduces errors, such as electronic transfer.

While no clinically significant systematic bias was detected, the potential for this bias remained given the volume of unlinked cases in each of the emergency care pathways.

The mean population data for the average Victorian ED presentation included all of the patient presentations for the respective time period, including those from the Referral Service who were sent to the emergency care pathways and presented in the ED. The presence of these cases in the 'average ED presentation' group will increase the overall ED suitability rate for this group. The impact would be negligible however with all Referral Service cases referred to the emergency care pathways only constituting $1.6 \%$ of the total ED workload if they had all presented at the ED during the study timeframe. Finally, 
the ED suitability measure was directly compared with the 'potentially avoidable GP-type presentations' despite their slight difference.

While the variation in secondary telephone triage system structure and functionality could not be addressed in this study, the research variables used were specifically selected to allow for similar methodological approaches, less vulnerable to personal opinion, to be used in future work. Using these methodological approaches, the findings may be somewhat limited in their broader generalisability, however they should be locally reproducible.

\section{CONCLUSION}

This study used linked ambulance and hospital data to analyse the appropriateness of the referral of cases for ED presentation following secondary telephone triage and provided a methodological approach that can be applied in future research. Overall, secondary telephone triage was able to appropriately identify many cases that were suitable for the ED and that would be admitted, at a rate higher than that of the average Victorian ED presentation. A small cohort of cases identified as suitable for alternative care pathways presented in the ED and were ED suitable. Further investigation is required beyond this study to ensure cases were not incorrectly triaged to the alternative care pathways and to optimise the suite of alternate pathways to ensure the right patient is being triaged to the right service.

Acknowledgements The staff from the Victorian Data Linkages unit at the Victorian Department of health who conducted the hospital data extraction and linkage to the prelinked Ambulance Victoria datasets.

Contributors KE: study conception, conducted the data linkage for the Ambulance Victoria datasets, analysed data and wrote the paper. KS: discussed core ideas, oversaw the data extraction, consulted on the data analysis and edited the paper. AM: discussed core ideas, consulted on the data analysis and edited the paper. JS: discussed core ideas to study, edited the paper. JS is Ms Eastwood's Primary PhD supervisor.

\section{Funding None declared.}

Competing interests $\mathrm{KE}$ is an intensive care paramedic and has previously worked as a call-taker with the Ambulance Victoria Referral Service (secondary telephone triage service). JE was the Chair of the Board of Ambulance Victoria. $\mathrm{KS}$ is the Manager of Research and Evaluation for Ambulance Victoria. AM has no competing interests.

Ethics approval Ethics approval was granted by the Monash University Human Research Ethics Committee (CF12/0547-2012000215) and the Ambulance Victoria Research Committee (R11-021).

Provenance and peer review Not commissioned; externally peer reviewed.

Data sharing statement № additional data are available.

Open Access This is an Open Access article distributed in accordance with the Creative Commons Attribution Non Commercial (CC BY-NC 4.0) license, which permits others to distribute, remix, adapt, build upon this work non-commercially, and license their derivative works on different terms, provided the original work is properly cited and the use is non-commercial. See: http://creativecommons.org/ licenses/by-nc/4.0/

(C) Article author(s) (or their employer(s) unless otherwise stated in the text of the article) 2017. All rights reserved. No commercial use is permitted unless otherwise expressly granted.

\section{REFERENCES}

1. Eastwood K, Morgans A, Smith K, et al. A novel approach for managing the growing demand for ambulance services by low-acuity patients. Aust Health Rev 2015.

2. Wrigley $\mathrm{H}$, George $\mathrm{S}, \mathrm{Smith} \mathrm{H}$, et al. Trends in demand for emergency ambulance services in Wiltshire over nine years: observational study. BMJ 2002;324:646-7.

3. Weaver MD, Moore CG, Patterson PD, et al. Medical necessity in emergency medical services transports. Am J Med Qual 2012;27:250-5.

4. Brown E, Sindelar J. The emergent problem of ambulance misuse. Ann Emerg Med 1993;22:646-50.

5. Chen JC, Bullard MJ, Liaw SJ. Ambulance use, misuse, and unmet needs in a developing emergency medical services system. Eur J Emerg Med 1996;3:73-8.

6. Ohshige K. Reduction in ambulance transports during a public awareness campaign for appropriate ambulance use. Acad Emerg Med 2008;15:289-93.

7. Fox C, Rodriguez C, McSwain NE, et al. EMT telephone triage. 1981;5:410-5.

8. Audit Commission for Local Authorities the National Health Service. A Life in the fast lane: value for money in emergency ambulance services: audit commission for local authorities and the national health service in England and Wales, 1998.

9. Richards JR, Ferrall SJ. Inappropriate use of emergency medical services transport: comparison of provider and patient perspectives. Acad Emerg Med 1999;6:14-20.

10. Brokaw J, Olson L, Fullerton L, et al. Repeated ambulance use by patients with acute alcohol intoxication, seizure disorder, and respiratory illness. Am J Emerg Med 1998;16:141-4.

11. Gratton MC, Ellison SR, Hunt J, et al. Prospective determination of medical necessity for ambulance transport by paramedics. Prehosp Emerg Care 2003;7:466-9.

12. Victorian Goverment. Working with paramedics to end the ambulance crisis. Department of Health. Melbourne: State Government Victoria, 2015.

13. National Association of E. M. S. Physicians, American College of Emergency Physicians Alternate ambulance transportation and destination. Ann Emerg Med 2001;38:616.

14. Hjälte L, Suserud BO, Herlitz J, et al. Why are people without medical needs transported by ambulance? A study of indications for prehospital care. Eur J Emerg Med 2007;14:151-6.

15. Uscher-Pines L, Pines J, Kellermann A, et al. Deciding to visit the emergency department for non-urgent conditions: a systematic review of the literature. The American journal of managed care 2013;19:47-59.

16. Ismail SA, Gibbons DC, Gnani S. Reducing inappropriate accident and emergency department attendances. a systematic review of primary care service interventions. 2013;63:e813-e20.

17. Schoenfeld EM, McKay MP. Weekend emergency department visits in Nebraska: higher utilization, lower acuity. J Emerg Med 2010;38:542-5.

18. Farion KJ, Wright M, Zemek R, et al. Understanding lowacuity visits to the pediatric emergency department. PLoS One 2015;10:e0128927.

19. Blunt I, Bardsley M, Dixon J. Trends in emergency admissions in England 2004-2009: is greater efficiency breeding inefficiency? The Nuffield Trust. 2010.

20. Snooks H, Wrigley H, George S, et al. Appropriateness of use of emergency ambulances. J Accid Emerg Med 1998;15:212-5.

21. Lowthian JA, Curtis AJ, Jolley DJ, et al. Demand at the emergency department front door: 10-year trends in presentations. Med J Aust 2012;196:128-32.

22. Nagree Y, Gosbell AD, Fatovich DM, et al. General practice patients form an insignificant part of the emergency department workload. Med J Aust 2012;197:619.

23. Schull MJ, Kiss A, Szalai JP. The effect of low-complexity patients on emergency department waiting times. Ann Emerg Med 2007;49 25764.

24. Tsai JC, Liang YW, Pearson WS. Utilization of emergency department in patients with non-urgent medical problems: patient preference and emergency department convenience. J Formos Med Assoc 2010;109:533-42.

25. Australian Institute of Health and Welfare. AloHa W, ed. Australian hospital statistics 2011-2012. Canberra: Australian Governement, 2013.

26. Fivaz C, Marshall G. Necessary components of a secondary telephonic medical triage system at 9-1-1. Utah, 2015.

27. Cunningham S. Getting the right outcome for "000" patients: Revising AV's operating model. Melbourne: Victorian Healthcare Association, 2016. http://www.vha.org.au/docs/sue-cunninghamfinal-presentation-ambulance-vic.pdf. 
28. Gardett I, Scott G, Clawson J, et al. 911 Emergency communication nurse triage reduces EMS patient costs and directs patients to highsatisfaction alternative point of care. Ann Emerg Dispatch Response 2015;3:8-13.

29. Lowthian JA, Cameron PA, Stoelwinder JU, et al. Increasing utilisation of emergency ambulances. Aust Health Rev 201135 63:7.

30. Dale J, Higgins J, Williams S, et al. Computer assisted assessment and advice for "non-serious" 999 ambulance service callers: the potential impact on ambulance despatch. Emerg Med J 2003;20:178-83.

31. Dale J, Williams S, Foster T, et al. Safety of telephone consultation for "non-serious" emergency ambulance service patients. Qual Saf Health Care 2004;13:363-73.

32. Studnek J. Thestrup L, Blackwell T, Bagwell B. Utilization of prehospital dispatch protocols to identify low-acuity patients. Prehosp Emerg Care 2012;16:204-9.

33. Smith WR, Culley L, Plorde M, et al. Emergency medical services telephone referral program: an alternative approach to nonurgent 911 calls. Prehosp Emerg Care 2001;5:174-80.

34. Crowther L, Williams R. Nurse interventions in ambulance commandand-control centres. Emerg Nurse 2009;17:22-5.

35. Turner J, Snooks H, Youren A, et al. The costs and benefits of managing some low priority 999 ambulance calls by NHS Direct nurse advisers. Final report for the National Coordinating Centre for NHS SDO R\&D The University of Sheffield. 2006.

36. Nagree Y, Ercleve TN, Sprivulis PC. After-hours general practice clinics are unlikely to reduce low acuity patient attendances to metropolitan Perth emergency departments. Aust Health Rev 2004;28:285-91.

37. Nguyen ND, Moore JB, Mclntosh NP, et al. Emergency department triage of low acuity patients to a Federally Qualified Health Center. $J$ Miss State Med Assoc 2013;54:280-3.

38. Ambulance Victoria. Referral Service Review: The case for expansion Doncaster. 2011. Report No: (Unpublished report) (accessed 22 Nov 2011)

39. Lowthian JA, Jolley DJ, Curtis AJ, et al. The challenges of population ageing: accelerating demand for emergency ambulance services by older patients, 1995-2015. Med J Aust 2011;194:574-8.

40. Eastwood K, Morgans A, Smith K, et al. Secondary triage in prehospital emergency ambulance services: a systematic review. Emerg Med J 2015;32:486-92.
41. Australian Bureau of Statistics. State and territory statistical indicators. Canberra: Australian Government, 2012. http://www.abs. gov.au/ausstats/abs@.nsf/Lookup/by+Subject/1367.0 2012 Main+ Features Estimated+Resident+Population 3.1. (accessed 29 Jan 2013).

42. McKesson Corp. McKesson San Francisco2013 (Care Enhance Call Center). http://www.mckesson.com/en us/McKesson.com/ About\%2BUs/Newsroom/Press\%2BReleases\%2BArchives/ 2001/McKesson\%2BCorporation\%2BAnnounces\%2BGeneral\% 2B\%2BAvailability\%2Bof\%2BNew\%2BCareEnhance\%2BCall\% 2BCenter\%2BSoftware.html.

43. Department of Health. Victorian Emergency Minimum Dataset (VEMD) User Manual. Melbourne: Department of Health, 2013:p. 105.

44. Department of Health. Victorian Admitted Episodes Dataset (VAED) user manual. 23rd ed. Melbourne: Department of Health, 2013:p. 38.

45. Australian Bureau of Statistics. Private Hospitals Australia 2011-12. Canberra: Australian Government, 2013. http://www.abs.gov.au/ ausstats/abs@.nsf/Lookup/0BC8E5C3636D11CFCA257BB8007 FDD96?opendocument. (accessed 13 Jun 2017)

46. Dusetzina S, Tyree S, Meyer A, et al. An overview of record linkage methods. Linking data for health services research: a framework and instructional guide.Maryland agency for healthcare research and quality. 2014.

47. Australian institute of Health and Welfare. Australian Hospital Statistics 2009-2010. Canberra: Australian Government, 2011.

48. Australian Institute of Health and Welfare. Australian Hospital Statistics 2010-2011. Canberra: Australian Government, 2012.

49. Blank L, Coster J, O'Cathain A, et al. The appropriateness of, and compliance with, telephone triage decisions: a systematic review and narrative synthesis. J Adv Nurs 2012;68:2610-21.

50. Australian Institute of Health and Welfare. Australian hospital statistics 2011-2012 Emergency department care. Canberra: AlHW, 2013

51. Australasian College for Emergency Medicine. Guidelines on the implementation of the Australasian triage scale in emergency department. West Melbourne: Australasian College for Emergency Medicine, 2013. Contract No: G24.

52. Corp IMB. IBM SPSS Statistics for Windowsed. 20.0. Armonk, NY: IBM Corp, 2011.

53. Cone DC, Benson R, Schmidt TA, et al. Field triage systems: methodologies from the literature. Prehosp Emerg Care 2004;8:130-7. 